\title{
Review of: "Helically arranged cross struts in azhdarchid pterosaur cervical vertebrae and their biomechanical implications"
}

Hiroaki Nakashima

Potential competing interests: The author(s) declared that no potential competing interests exist.

Although this paper shows interesting result of cervical vertebrae of a rare animal, little is known about azhdarchid pterosaurs, and then more discussion about similar animals is mandatory. Furthermore. this model was made based on the fossil, and the validity of the 3D model might have a gap between a living body and this model. 